\title{
Antimicrobial Activities of Five Strains Of Lactococcus Isolated from Beef Against Indicator Organisms of Public Health Significance
}

\author{
Olusegun Ayodele Olaoye*
}

Department of Food Science and Technology, Michael Okpara University of Agriculture, Umudike, Abia State, Nigeria A R T I C LE IN F O

Article history:

Received 27 June 2016

Accepted 28 September 2016

Available online, ISSN: 2148-127X

Keywords:

Lactococcus

Antimicrobial agents

Spoilage and pathogenic organisms

Antimicrobial activities

Public health significance

Corresponding Author:

E-mail: olaayosegun@yahoo.com

A B S T R A C T

Five strains of Lactococcus, including L. garviae K2, L. piscium SU4, L. lactis subsp. cremoris E22, L. plantarum $\mathrm{L} 7$ and L. lactis subsp. hordinae E91 were screened for production of antimicrobial agents. The strains were also analysed for antimicrobial activities against spoilage and pathogenic organisms, including Staphylococcus aureus, Salmonella Typhimurium, Escherichia coli, Yersinia enterocolitica, Pseudomonas aeruginosa, Klebsiella pneumoniae, Listeria monocytogenes, Bacillus cereus and Pseudomonas fluorescens. Result revealed that L. piscium SU4, L. plantarum L7 and L. lactis subsp. cremoris E22 had lactic acid production (g/10 ${ }^{7}$ colony forming units, CFU) of 7.23, 7.20 and 7.19. The value of 6.83 recorded as lactic acid produced by L. garviae K2 was significantly different from those obtained for others. The highest acetic acid production $\left(3.55 \mathrm{~g} / 10^{7} \mathrm{CFU}\right)$ was recorded for L. garviae $\mathrm{K} 2$ while L. piscium SU4 had the lowest $\left(2.99 \mathrm{~g} / 10^{7} \mathrm{CFU}\right)$. L. lactis subsp. hordinae E91 had diacetyl production of $71.99\left(\mu \mathrm{g} / 10^{7} \mathrm{CFU}\right)$, which was higher than those recorded for other Lactococcus strains. Test for antimicrobial activities showed that Escherichia coli NCTC 86, Yersinia enterocolitica NCTC 10460, Pseudomonas aeruginosa NCIMB 10848, Bacillus cereus NCTC 21113 and Pseudomonas flourescens recorded higher susceptibilities to the antimicrobial action of the Lactococcus strains than others; zones of inhibition of $5 \mathrm{~mm}$ and above were recorded for the indicator organisms compared to lower values $(<5 \mathrm{~mm})$ obtained for others. Listeria monocytogenes also showed medium susceptibility (zones of inhibition $<5 \mathrm{~mm}$ ) to the antimicrobial activities of the Lactococcus strains. It was concluded that the Lactococcus strains could be effective in the control of spoilage and pathogenic organisms; their antagonism recorded against Listeria monocytogenes, E. coli, Salmonella Typhimurium and Staphylococcus aureus may be of public health significance.

\section{Introduction}

The lactic acid bacteria (LAB) comprise a group of Gram positive, non-sporulating, cocci or rods, and are catalases lacking organisms. They produce lactic acid as the major end product during the fermentation of carbohydrates, and only grow in complex media where fermentable carbohydrates and higher alcohols are used as an energy source, mainly to form lactic acid (Olaoye and Ntuen, 2011). Homofermentative LAB degrade hexoses to lactic acid (lactate), whereas heterofermentative LAB degrade hexoses to lactate and additional products such as acetic acid (acetate), ethanol, $\mathrm{CO} 2$, formic acid (formate), or succinic acid (succinate). LAB is widespread in most ecosystems and is found in soil, water, plants, and animals. They are responsible for many food fermentation processes, but they are also commonly found on nonfermented foods such as dairy products, meat products, seafood, fruits, vegetables, cereals, sewage, and in the genital, intestinal, and respiratory tracts of humans and animals. LAB are widely used as protective cultures in the food industry for the production of fermented foods, including dairy (yogurt, cheese), meat (sausages), fish, cereals (bread and beverages such as beer), fruit (malolactic fermentation processes in wine production) and vegetables such as sauerkraut, kimchi and silage (Olaoye and Ntuen, 2011).

LAB is expected to produce of lactic acid from carbohydrate sources rapidly in adequate concentrations. The primary antimicrobial effect exerted by LAB is the production of lactic acid resulting in reduction of $\mathrm{pH}$; the levels of production of organic acids by LAB depend on the species or strain which are normally affected by growth medium composition and conditions (Lindgren and Dobrogosz, 1990). In addition, they produce various low-molecular-mass compounds such as hydrogen peroxide $\left(\mathrm{H}_{2} \mathrm{O}_{2}\right)$, carbon dioxide $\left(\mathrm{CO}_{2}\right)$, diacetyl (2,3butanedione), uncharacterized compounds and highmolecular-mass compounds like bacteriocins. All of these can antagonize the growth of some spoilage and pathogenic bacteria in foods and have been explored in the control of most unwanted organisms, but their production is variable among strains of LAB (Olaoye and Onilude, 2011). Many LAB produce a range of 
antimicrobial substances that are generally active towards other LAB (especially closely related strains) and food borne pathogens such as Listeria monocytogenes, Staphylococcus aureus, Clostridium perfringens and Bacillus cereus (Noonpakdee et al., 2003). LAB comprise of many genera including Lactobacillus, Lactococcus, Leuconostoc, Pediococcus and Streptococcus (Olaoye, 2014). The genus Lactococcus comprises many species, and they have been isolated from several food sources, especially dairy and meat.

The present study reports the production of some antimicrobial agents by some strains of Lactococcus which have been isolated from beef in a previous study (Olaoye, 2014). Their antimicrobial activities against known spoilage organisms of food and pathogenic organisms are also reported, with the objective of proposing suitable strains for use in the biopreservation of food as a results of the generally regarded as safe (GRAS) status of LAB.

\section{Materials and Methods}

Source of Lactococcus Strains, Spoilage/Pathogenic Organisms and Culture Conditions

The Lactococcus strains used in the present study consisted of five microbial isolates that have been isolated and identified from Nigerian beef in a previous study (Olaoye, 2014). The indicator spoilage and pathogenic organisms used in challenging the antimicrobial activities of the Lactococcus strains were obtained from the Food Microbiology laboratory of the Department of Microbiology, University of Ibadan (Table 1). They include Staphylococcus aureus, Salmonella Typhimurium, Escherichia coli NCTC 86, Yersinia enterocolitica NCTC 10460, Pseudomonas aeruginosa (NCIMB 10848), Klebsiella pneumoniae U11468, Listeria monocytogenes NCTC 11994, Bacillus cereus NCTC 21113 and Pseudomonas fluorescens.
The optimal growth temperatures and culture medium of the different organisms are shown in Table 1.

Measurement of Technological Properties Contributing to Antimicrobial Activities of the Lactococcus Strains

Organic acids (lactic and acetic acids): The slightly modified HPLC method described by Olaoye et al. (2008) was used to measure the organic acids production by the Lactococcus strains in vitro assay. This involved the use of an inoculum $(100 \mu \mathrm{l})$ each of the Lactococcus strains, representing approximately $10^{6} \mathrm{CFU} / \mathrm{ml}$ in $30 \mathrm{ml}$ sterile M17 broth in universal bottle (Inoculum was taken from cultured strains previously grown in M17 broth at $30^{\circ} \mathrm{C}$ for $24 \mathrm{~h}$, with optical density adjusted to same value each); the universal bottles were incubated at $30^{\circ} \mathrm{C}$ in a shaking incubator $(200 \mathrm{rpm})$ for $24 \mathrm{~h}$. Starting from $0 \mathrm{~h}$, samples were taken every $6 \mathrm{~h}$ during incubation measurement of organic acids. Samples $(15 \mathrm{ml})$ were centrifuged at 3,500 x $\mathrm{g}$ for $15 \mathrm{~min}$, and the cell free supernatants (CFS) were obtained and filter sterilized, using $0.2 \mu \mathrm{m}$ syringe filter (Sartorius AG 37070 Goettingen, Germany). Uninoculated M17 broth, similarly treated as other samples, was used to set baseline for measurement of the organic acids. Standard concentrations (g/l) lactic and acetic acids were prepared and analysed by HPLC, results of which were used to plot standard curves from which the concentrations of the acids in the samples were measured. Concentrations of lactic and acetic acids were normalized as g per $10^{7} \mathrm{CFU}$.

The HPLC system and chromatographic conditions used were same as previously described (Olaoye et al., 2008). The HPLC system used consisted of LC-10ADVP pump (Shimadzu, UK), equipped with injection valve of $20 \mu 1$ capacity; UV detector (SpectroMonitor 3000, LDC/Milton Roy, Florida, USA); Data recorder (Picolog for windows, Release 5.12.1, St Neots, UK); C18 analytical column (a reverse phase Techsphere ODS-2 5U, 250mm length, 4.6mm internal diameter).

Table 1 Lactococcus strains and other microorganism used, sources and growth conditions

\begin{tabular}{l|lll}
\hline Name & Media & Temp $\left({ }^{\circ} \mathrm{C}\right)$ & Source \\
\hline Lactococcus garviae K2 & M17 & 30 & Olaoye, 2014 \\
L. piscium SU4 & M17 & 30 & Olaoye, 2014 \\
L. lactis subsp. cremoris E22 & M17 & 30 & Olaoye, 2014 \\
L. lactis subsp. hordinae E91 & M17 & 30 & Olaoye, 2014 \\
L. plantarum L7 & M17 & 30 & Olaoye, 2014 \\
Staphylococcus aureus & BHI & 37 & Food Microbiology laboratory, Dept of \\
& & & Microbiology, University of Ibadan, Nigeria \\
Salmonella Typhimurium & BHI & 37 & “ \\
Escherichia coli NCTC 86 & BHI & 37 & “ \\
Yersinia enterocolitica NCTC 10460 & BHI & 37 & “ \\
Pseudomonas aeruginosa (NCIMB 10848) & NB & 30 & “ \\
Klebsiella pneumoniae U11468 & BHI & 37 & “ \\
Listeria monocytogenes NCTC 11994 & BHI & 30 & “ \\
Bacillus cereus NCTC 21113 & NB & 37 & NB \\
Pseudomonas fluorescens & NB & 30 & \\
\hline
\end{tabular}

M17, growth medium for cultivation of Lactococcus; BHI, brain heart infusion; NB, nutrient broth 
Analysis was performed at ambient temperature with a mobile phase consisting of $0.02 \mathrm{M} \mathrm{NaH}_{2} \mathrm{PO}_{4}$ (adjusted to $\mathrm{pH} 2.55 \pm 0.02$ with $\left.\mathrm{H}_{3} \mathrm{PO}_{4}\right)$ and methanol $(98 \% \mathrm{v} / \mathrm{v})$ and delivered at a flow rate of $1 \mathrm{ml} / \mathrm{min}$. The samples were detected by their UV absorbance monitoring at $220 \mathrm{~nm}$ with sensitivity setting of 0.002 absorbance units full scale (AUFS). The pressure of pump was $13.5 \pm 0.5 \mathrm{Mpa}$. Sample injections into HPLC were made using a $50 \mu \mathrm{l}$ stainless steel syringe holder, with a maximum of $20 \mu \mathrm{l}$ being injected into the injection valve, while the remaining was collected as waste through the waste outlet.

Diacetyl: Measurement of diacetyl (DA) production by the Lactococcus strains was accomplished by the use of headspace analysis and gas chromatography-mass spectrometry (GC-MS) as described by Olaoye et al. (2008). Sample preparation was done as described for the organic acids. A known concentration of pure DA was added into the CFS and recovery attempts were then made, a recovery concentration of DA above $100 \%$ was assumed to be due to the quantity of DA in the original CFS and added concentration of the pure compound. Preparation of standard concentrations of DA was made in blank M17, analysed by GC and the results were used to plot a standard graph, from which the concentrations of DA in the samples were measured. Blank M17 broth was used to set a baseline for measuring DA.

Concentrations of DA were expressed as $\mu \mathrm{g} / 10^{7} \mathrm{CFU}$.

Antimicrobial Activities of the Lactococcus Strains Against Indicator Organisms

Detection of antagonism of the Lactococcus strains against indicator organism was done by placing a drop $(20 \mu \mathrm{l})$ of BHI broth (in which each of the strains has been grown for $18 \mathrm{~h}$ ) on petri dish plates already inoculated with indicator organisms. Plates were inoculated at $30^{\circ} \mathrm{C}$ for $24 \mathrm{~h}$ after which they were examined for occurrence of antagonism (Figure 1).

The paper disc assay method (Gurira and Buys, 2006) was used for measuring zones of inhibition (Figure 2). A sterile filter paper disc (Whatman AA, $6 \mathrm{~mm}$, Fisher Scientific, UK) was soaked in CFS for $30 \mathrm{~min}$, and then applied on plates previously seeded with BHI or Nutrient broth (with $0.7 \%$ agar) containing $50 \mu \mathrm{l}$ of indicator organisms. The plates were incubated overnight at $30^{\circ} \mathrm{C}$ for $24 \mathrm{~h}$ and zones of inhibition were measured. Clear zones extending for $1 \mathrm{~mm}$ or more were considered as positive for inhibition (Noordiana et al., 2013).

Antagonism by the Lactococcus strains against the indicator organisms was also detected by adding $500 \mu \mathrm{l}$ of CFS of the strains to BHI broth (already inoculated with indicator organism) at $5 \mathrm{~h}$ during incubation. The optical density (OD) of the indicator organism was monitored during incubation, before and after addition of CFS, at $580 \mathrm{~nm}$ to detect likely presence of antagonism (Noordiana et al., 2013; Olaoye, 2015).

\section{Statistical Analysis}

The data obtained were analysed using the means of three replicates of each sample. Means were separated and analysed using the $t$-test in data analysis functionality of Microsoft Excel 2010 to determine differences. Significant differences between samples were determined at $\mathrm{P}<0.05$.

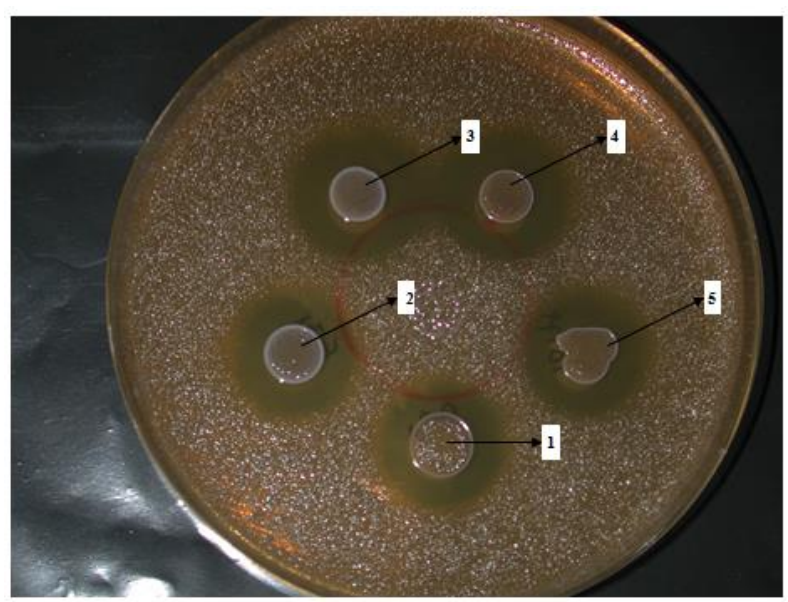

Figure 1 Antagonistic activities of the Lactococcus strains against Yersinia enterocolitica NCTC 10460

1, Lactococcus garviae K2; 2, L. piscium SU4; 3, L. lactis subsp. cremoris E22; 4, L. lactis subsp hordinae E91; 5, L. plantarum $\mathrm{L} 7$

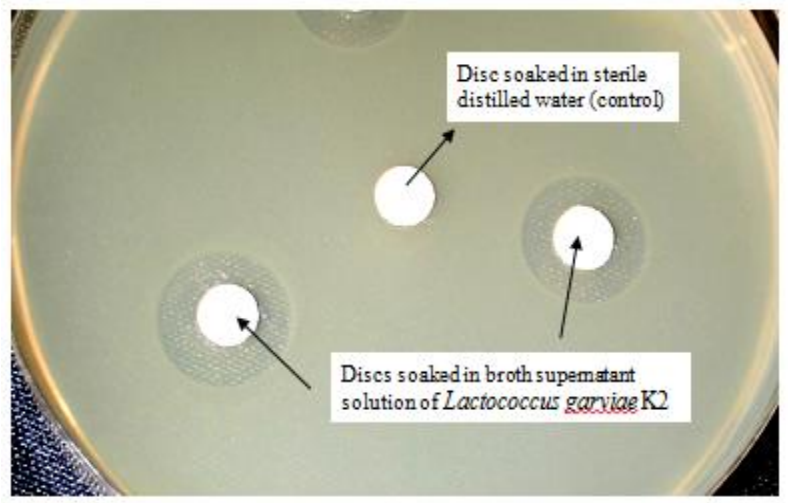

Figure 2 In vitro inhibition of Lactococcus garviae K2 against Klebsiella pneumoniae U11468, using the disc assay method.

\section{Results and Discussion}

The strains of Lactococcus used in this study were isolated and identified in a previous report (Olaoye, 2014). The indicator spoilage and pathogenic organisms used in challenging the antimicrobial activities of the Lactococcus strains were obtained from the Food Microbiology laboratory of the Department of Microbiology, University of Ibadan (Table 1). They include Staphylococcus aureus, Salmonella Typhimurium, Escherichia coli NCTC 86, Yersinia enterocolitica NCTC 10460, Pseudomonas aeruginosa (NCIMB 10848), Klebsiella pneumoniae U11468, Listeria monocytogenes NCTC 11994, Bacillus cereus NCTC 21113 and Pseudomonas fluorescens; only one strain each of the different organisms were used in this study. 
Figure 1 shows the result of in vitro test for antimicrobial activities of the Lactococcus strains against Yersinia enterocolitica NCTC 10460, using the spot on agar method. All the strains displayed antagonism against the indicator organism in vitro, suggesting that they may be able to control its growth in food. In Figure 2, Lactococcus garviae $\mathrm{K} 2$ was shown to display antimicrobial activity against Klebsiella pneumoniae U11468 with the use of disc method. The disc method was used in measuring zones of inhibition of the Lactococcus strains against the different indicator organisms.

Presented in Table 2 are the concentrations of organic acids (lactic and acetic acids) and diacetyl produced by the Lactococcus strains in growth medium at $24 \mathrm{~h}$ of incubation. Results revealed that all the strains, with the exception of $L$. garviae $\mathrm{K} 2$, had lactic concentration higher than $7.00 \mathrm{~g} / 10^{7} \mathrm{CFU}$ and no significant difference $(\mathrm{P}>0.05)$ was recorded in their values. However, concentration of $6.83 \mathrm{~g} / 10^{7} \mathrm{CFU}$ was obtained for $L$. garviae $\mathrm{K} 2$, the value which was significantly lower $(\mathrm{P}<0.05)$ than those recorded for other strains. Production of acetic acid (3.55 g/10 $\left.{ }^{7} \mathrm{CFU}\right)$ was recorded for $L$. garviae $\mathrm{K} 2$, and this was significantly higher than those obtained for other Lactococcus strains $(\mathrm{P}<0.05)$. Production of organic acids has been noted as a key feature which contributes to antimicrobial activities of lactic acid bacteria against unwanted microorganism; and this has been reported in many research investigations (Sahnouni et al., 2014; Olaoye, 2014; Olaoye, 2015). Concentrations of lactic and acetic acids recorded for the Lactococcus strains were similar to those reported for other types of LAB in similar studies (Olaoye and
Onilude, 2011; Olaoye, 2014). Olaoye and Onilude (2011) recorded lactic acid concentrations of between 5.55 to $7.99 \mathrm{~g} / 10^{7} \mathrm{CFU}$ for Pediococcus strains of LAB during $24 \mathrm{~h}$ of growth, the values which were supportive of those recorded in the present study. The research workers also reported acetic acid concentrations of between 2.88 and $6.00 \mathrm{~g} / 10^{7} \mathrm{CFU}$ for the LAB strains, which were also in support of those recorded in the present study. Furthermore, Olaoye (2014) reported acetic acid values of 2.31 to $4.22 \mathrm{~g} / 10^{7} \mathrm{CFU}$ for some Lactococcus strains within $24 \mathrm{~h}$ of growth, and this was consistent with those recorded in the present report. The concentration of the organic acids was observed to correlate with antimicrobial activities of the Lactococcus strains against many of the spoilage and pathogenic organisms that were tested as shown in Table 3; this was discussed later in this section.

Concentrations of diacetyl produced by the Pediococcus strains ranged between 31.25 and 71.99 $\mu \mathrm{g} / 10^{7} \mathrm{CFU}$, with L. lactis subsp. hordinae E91 having the highest value and the lowest was recorded for L. lactis subsp. cremoris E22. Diacetyl has been noted as a metabolite produced by LAB which contributes to their antimicrobial activities against spoilage and pathogenic organisms (Adejumo, 2014; Emerenini et al., 2014; Olaoye, 2015). Production of diacetyl by the Lactococcus strains in the present study may therefore be desirable towards control of unwanted organisms, especially spoilage and pathogenic types, in food products. As observed for the organic acids, concentration of diacetyl had direct correlation with the antimicrobial activities of the different strains against the indicator organisms and this was subsequently discussed in this section

Table 2 Measured technological properties contributing to antimicrobial activities of the Lactococcus strains

\begin{tabular}{|c|c|c|c|c|c|c|c|c|c|c|}
\hline \multirow{3}{*}{ Property } & \multicolumn{10}{|c|}{ Lactococcus strains } \\
\hline & \multicolumn{2}{|c|}{ L. garviae $\mathrm{K} 2$} & \multicolumn{2}{|c|}{ L. piscium SU4 } & \multicolumn{2}{|c|}{$\begin{array}{l}\text { L. lactis } \text { subsp } \\
\text { cremoris } \text { E22 }\end{array}$} & \multicolumn{2}{|c|}{$\begin{array}{l}\text { L. lactis subsp } \\
\text { hordinae E91 }\end{array}$} & \multicolumn{2}{|c|}{ L. plantarum L7 } \\
\hline & \multicolumn{2}{|c|}{ value SD } & \multicolumn{2}{|c|}{ value SD } & \multicolumn{2}{|c|}{ value SD } & \multicolumn{2}{|c|}{ value SD } & \multicolumn{2}{|c|}{ value SD } \\
\hline Lactic acid (g/10 $\mathrm{CFU})$ & $6.83^{b}$ & 2.37 & $7.23^{\mathrm{a}}$ & 2.21 & $7.19^{\mathrm{a}}$ & 1.04 & $7.11^{\mathrm{a}}$ & 2.36 & $7.20^{\mathrm{a}}$ & 1.04 \\
\hline Acetic acid (g/10 $\mathrm{CFU})$ & $3.55^{\mathrm{a}}$ & 1.03 & $2.99^{\mathrm{b}}$ & 0.36 & $3.15^{\mathrm{b}}$ & 0.75 & $3.24^{\mathrm{b}}$ & 0.79 & $3.20^{\mathrm{b}}$ & 1.20 \\
\hline Diacetyl $\left(\mu \mathrm{g} / 10^{7} \mathrm{CFU}\right)$ & $45.32^{c}$ & 6.25 & $36.36^{\mathrm{d}}$ & 7.26 & $31.25^{\mathrm{d}}$ & 5.45 & $71.99^{\mathrm{a}}$ & 12.29 & $58.43^{\mathrm{b}}$ & 7.32 \\
\hline
\end{tabular}

Values are means of three replicates; $\mathrm{CFU}$, colony forming unit; SD, standard deviation; Values with different superscript letters across rows are significantly different $(\mathrm{P}<0.05)$.

Table 3 Zones of inhibition ( $\mathrm{mm}$ ) of the Lactococcus strains against spoilage and pathogenic organisms

\begin{tabular}{|c|c|c|c|c|c|}
\hline \multirow{2}{*}{ Spoilage and Pathogenic Organisms } & \multicolumn{5}{|c|}{ Lactococcus strains } \\
\hline & $\mathrm{K} 2$ & SU4 & E22 & E91 & L7 \\
\hline Staphylococcus aureus & $1.5^{\mathrm{b}}$ & $1.5^{\mathrm{b}}$ & $1.5^{\mathrm{b}}$ & $1.5^{\mathrm{b}}$ & $2.5^{\mathrm{a}}$ \\
\hline Samonella Typhimurium & $2.0^{\mathrm{c}}$ & $3.5^{\mathrm{b}}$ & $5.0^{\mathrm{a}}$ & $5.0^{\mathrm{a}}$ & $3.5^{\mathrm{b}}$ \\
\hline Escherichia coli NCTC 86 & $6.0^{\mathrm{b}}$ & $5.0^{\mathrm{b}}$ & $10.0^{\mathrm{a}}$ & $12.0^{\mathrm{a}}$ & $12.0^{\mathrm{a}}$ \\
\hline Yersinia enterocolitica (NCTC 10460) & $15.5^{\mathrm{a}}$ & $16.0^{\mathrm{a}}$ & $15.0^{\mathrm{a}}$ & $15.0^{\mathrm{a}}$ & $15.5^{\mathrm{a}}$ \\
\hline Pseudomonas aeruginosa (NCIMB 10848) & $18.0^{\mathrm{b}}$ & $20.0^{\mathrm{a}}$ & $21.0^{\mathrm{a}}$ & $17.0^{\mathrm{b}}$ & $16.0^{\mathrm{b}}$ \\
\hline Klebsiella pneumoniae U11468 & $1.5^{\mathrm{b}}$ & $2.0^{\mathrm{a}}$ & $2.0^{\mathrm{a}}$ & $1.5^{\mathrm{b}}$ & $2.0^{\mathrm{a}}$ \\
\hline Listeria monocytogenes (NCTC 11994) & $2.0^{\mathrm{a}}$ & $2.0^{\mathrm{a}}$ & $2.5^{\mathrm{a}}$ & $2.0^{\mathrm{a}}$ & $2.0^{\mathrm{a}}$ \\
\hline Bacillus cereus NCTC 21113 & $5.0^{\mathrm{a}}$ & $5.5^{\mathrm{a}}$ & $5.5^{\mathrm{a}}$ & $5.5^{\mathrm{a}}$ & $6.0^{\mathrm{a}}$ \\
\hline Pseudomonas flourescens & $12.0^{\mathrm{b}}$ & $14.0^{\mathrm{a}}$ & $15.0^{\mathrm{a}}$ & $10.0^{\mathrm{b}}$ & $11.0^{\mathrm{b}}$ \\
\hline
\end{tabular}




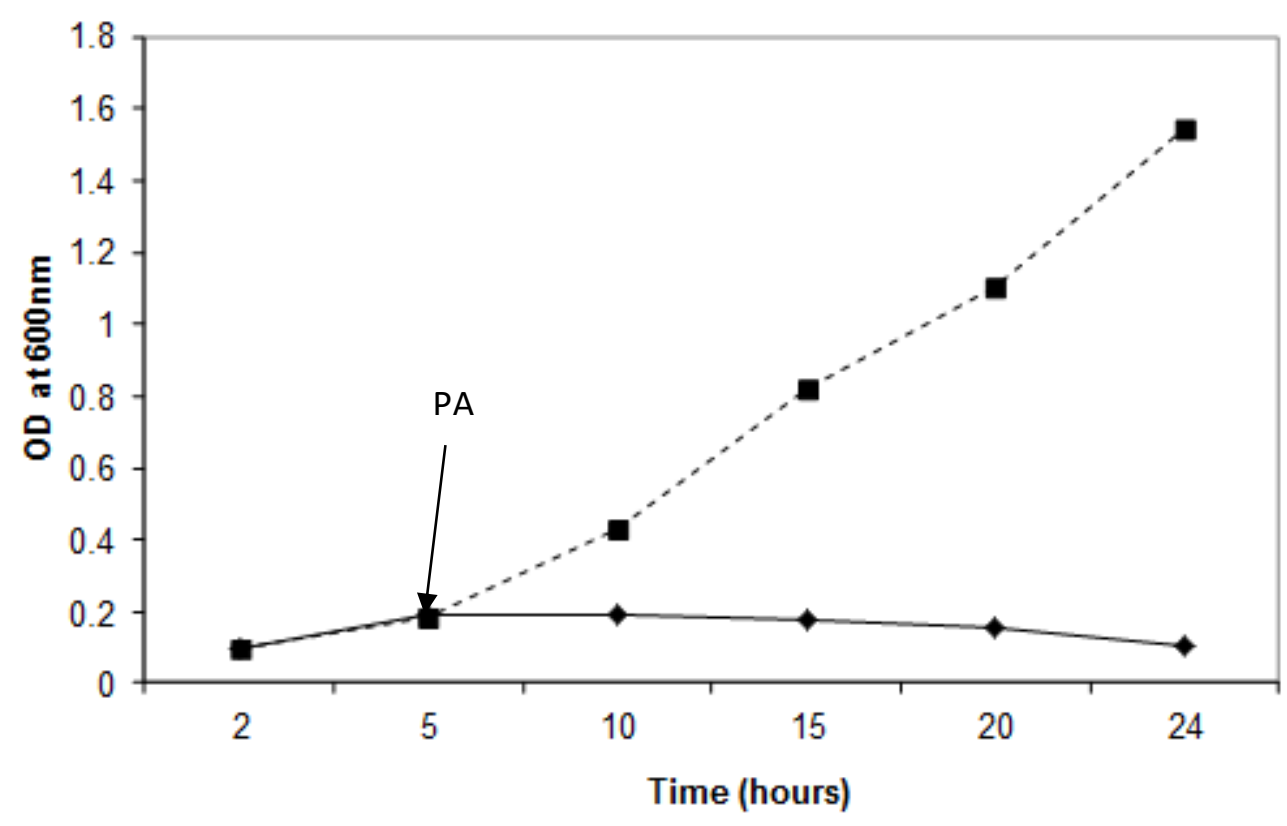

Figure 3 Effect of addition of CFS of Lactococcus garviae K2 on optical density (OD) of Klebsiella pneumoniae U11468 during growth; '-- - - ', OD of K. pneumoniae U11468 (no addition of L. garviae K2); '—^—', OD of K. pneumoniae U11468 after addition of L. garviae K2; PA, point of addition of broth supernatant solution of Lactococcus garviae $\mathrm{K} 2$

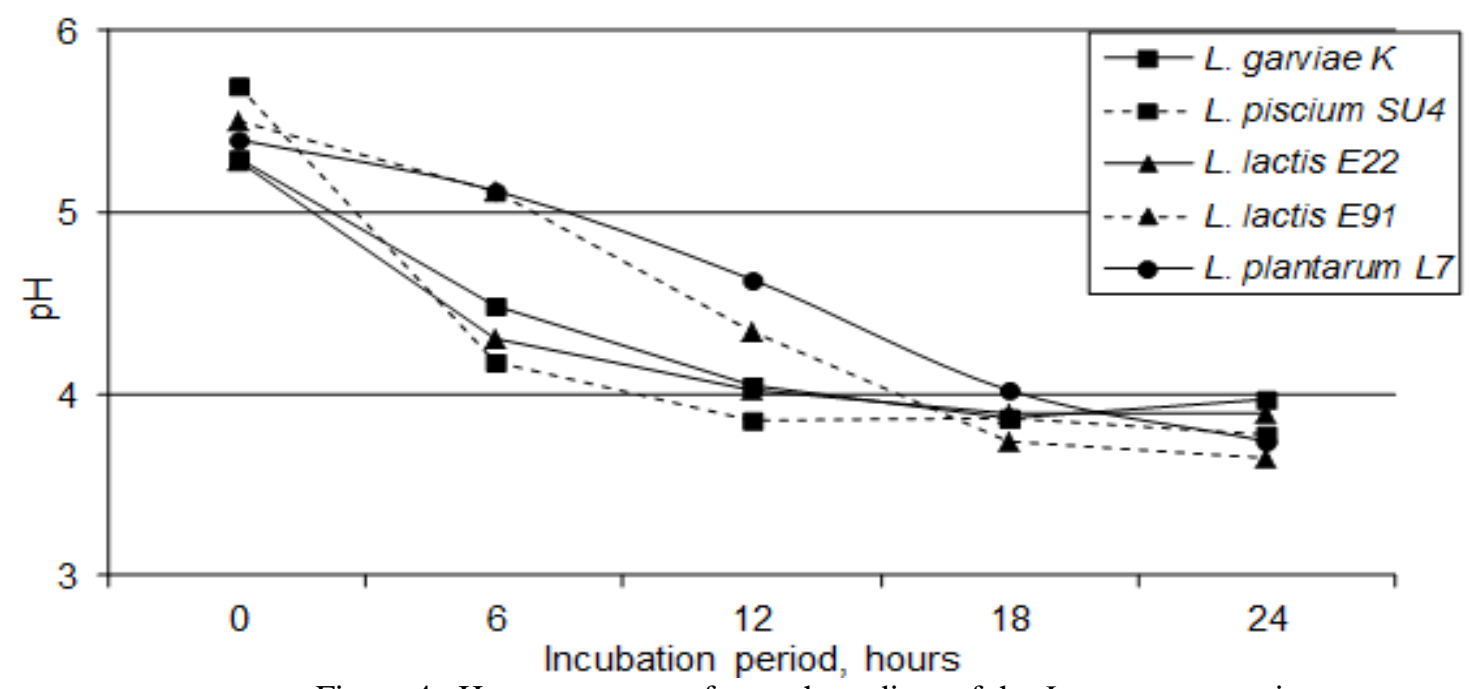

Figure $4 \mathrm{pH}$ measurement of growth medium of the Lactococcus strain

The zones of inhibitions produced by the Lactococcus strains against the spoilage and pathogenic organisms during challenge tests in vitro are shown in Table 3. Among the indicator organisms, Escherichia coli NCTC 86, Yersinia enterocolitica NCTC 10460, Pseudomonas aeruginosa NCIMB 10848, Bacillus cereus NCTC 21113 and Pseudomonas flourescens showed higher susceptibilities to the antimicrobial activities of the Lactococcus strains; zones of inhibition of $5 \mathrm{~mm}$ and above were recorded against them while $5 \mathrm{~mm}$ and below were obtained for other indicator organisms. In support of the results of the present study, Adejumo (2014) and Khandare and Patil (2015) reported successful inhibition by some LAB strains against Staphylococcus aureus,
Samonella Typhimurium and Escherichia coli. Sahnouni et al. (2014) and Khandare and Patil (2015) also noted inhibition of Pseudomonas aeruginosa by LAB in separate studies, thus further corroborating the result of the present study. In some research investigations, the abilities of LAB to control Listeria monocytogenes were reported by Olaoye and Onilude (2010), Olaoye et al. (2011) and Olaoye (2015); these reports justify the antimicrobial activities of the Lactococcus strains in the present study.

In this study, the antimicrobial activities of the Lactococcus strains recorded against Staphylococcus aureus, Samonella Typhimurium, Escherichia coli, Yersinia enterocolitica and Listeria monocytogenes may 
be of public health significance because they have been reported to be pathogenic in nature (Ammor et al., 2006; Sahnouni et al., 2014; Khandare and Patil, 2015). Among other factors, production of organic acids and diacetyl by the Lactococcus strains may have contributed to their antimicrobial action against the indicator organisms, as earlier noted.

Confirmation that antimicrobial activities of the Lactococcus strains may be due to the extracellular metabolites produced by them in growth medium was carried out by the addition of the CFS of the strains into medium containing indicator organisms during growth. A typical graph of the measurement of their optical densities (OD) during growth, before and after addition of CFS of the Lactococcus strains is presented in Figure 3. From the graph, it was observed that OD of the indicator organisms decreased shortly after the addition of CFS compared with control without CFS; a difference of up to 1.0 unit in OD was recorded between them. This further justified the assertion that the antimicrobial activities of LAB were as a result of extracellular metabolites produced by them in growth medium, including organic acids and diacetyl (Ammor and Mayo, 2007; Olaoye and Onilude, 2011; Adejumo, 2014; Khandare and Patil, 2015).

$\mathrm{pH}$ determination in growth medium of the Lactococcus strains indicated that decrease in values was observed during growth (Figure 4); values generally decreased below 4.0 at $24 \mathrm{~h}$ of incubation in growth medium. The decrease in $\mathrm{pH}$ levels during growth may be attributed to production of organic acids by the Lactococcus strains. The $\mathrm{pH}$ values recorded for the different strains correlated directly with the concentration of organic acids produced by them; the organic acids produced by the Lactococcus strains were lower than 1 $\mathrm{g} / 107 \mathrm{CFU}$ at 6-9 $\mathrm{h}$ of incubation, during which period $\mathrm{pH}$ was higher than 4.0. Organic acid concentrations generally increased during incubation, and this prompted corresponding decrease in $\mathrm{pH}$. Lowering of $\mathrm{pH}$ in food products due to growth of LAB is an important factor in the control of undesirable microorganisms (Kaban and Kaya, 2006). The reduction in $\mathrm{pH}$ recorded in the growth medium may also have contributed to the antimicrobial activities of the Lactococcus strains against the indicator organisms during the in vitro assay.

This study concluded that the antimicrobial action of the Lactococcus strains were effective in controlling some pathogenic and spoilage organisms in vitro. The antimicrobial actions recorded against Listeria monocytogenes, Escherichia coli, Salmonella Typhimurium and Staphylococcus aureus may be of public health significance as a result of their ability to cause diseases. It was also concluded that certain metabolites produced by the strains, especially organic acids and diacetyl, contributed to their antagonistic actions against the indicator organisms. Though the Lactococcus strains displayed antimicrobial activities against some spoilage and pathogenic organisms in vitro, it may be useful to evaluate their performance in food in situ in future studies.

\section{References}

Adejumo TO. 2014. Antimicrobial activity of lactic acid bacteria isolated from fermented milk products. African Journal of Food Science, 8: 490-496.

Ammor MS, Mayo B. 2007. Selection criteria for lactic acid bacteria to be used as functional starter cultures in dry sausage production: An update. Meat Science, 76: 138-146.

Ammor S, Tauveron G, Dufour E, Chevallier I. 2006. Antimicrobial activity of lactic acid bacteria against spoilage and pathogenic bacteria isolated from the same meat small-scale facility 1Screening and characterization of the antimicrobial compounds. Food Control, 17: 454-461.

Emerenini EC, Afolabi OR, Okolie PI, Akintokun AK. 2014. In vitro studies on antimicrobial activities of lactic acid bacteria isolated from fresh vegetables for biocontrol of tomato pathogens. British Microbiology Research Journal, 4(3): 351-359.

Gurira OZ, Buys EM. 2005. Characterization and antimicrobial activity of Pediococcus species isolated from South African farm-style cheese. Food Microbiology, 22: 159-168.

Kaban G, Kaya M. 2006. Effect of starter culture on growth of Staphylococcus aureus in sucuk. Food Control, 17: 797-801.

Khandare SS, Patil SD. 2015. Inhibitory activity of Lactic acid bacteria against isolated pathogens and spoilage organisms associated with fresh meat. International Journal of Current Microbiology and Applied Science, 2: 128-135.

Lindgren SE, Dobrogosz WJ. 1990. Antagonistic activities of lactic acid bacteria in food and feed fermentations. FEMS Microbiology Reviews, 7: 149-163.

Noonpakdee W, Santivarangkna C, Jumriangrit P, Sonomoto K, Panyim S. 2003. Isolation of nisin-producing Lactococcus lactis WNC 20 strain from nham, a traditional Thai fermented sausage. International Journal of Food Microbiology, 81: 137-145.

Noordiana N, Fatimah AB, Mun AS. 2013. Antibacterial agents produced by lactic acid bacteria isolated from Threadfin Salmon and Grass Shrimp. International Food Research Journal, 20: 117-124.

Olaoye OA, Onilude AA, Dodd CER. 2008. Identification of Pediococcus spp. from beef and evaluation of their lactic acid production in varying concentrations of different carbon sources. Advances in Natural and Applied Sciences, 2: 197-207.

Olaoye OA. 2014. Technological properties of Lactococcus lactis subsp. lactis $\mathrm{I} 23$ isolated from Nigerian beef and characterization of bacteriocin produced by it. British Biotechnology Journal, 4(7): 829-845.

Olaoye OA. 2015. Molecular characterization of selected strains of lactic acid bacteria isolated from Nigerian beef and evaluation of their antimicrobial properties against food borne/spoilage microorganisms. AASCIT Journal of Bioscience, 1(1): 1-8.

Olaoye OA, Onilude AA. 2011. Quantitative estimation of antimicrobials produced by Lactic Acid Bacteria isolated from Nigerian beef bacteria. International Food Research Journal, 18: 1104-1110.

Olaoye OA, Onilude AA. 2010. Investigation on the potential use of biological agents in the extension of fresh beef in Nigeria. World Journal of Microbiology and Biotechnology, 26: 1445-1454.

Olaoye OA, Onilude AA, Idowu OA. 2011. Microbiological profile of goat meat inoculated with lactic acid bacteria cultures and stored at $30^{\circ} \mathrm{C}$ for 7 days. Food and Bioprocess Technology, 4: 312-319.

Olaoye OA, Onilude AA. 2011. Quantitative estimation of antimicrobials produced by Lactic Acid Bacteria isolated from Nigerian beef bacteria. International Food Research Journal, 18: 1104-1110.

Olaoye OA, Ntuen IG. 2011. Spoilage and preservation of meat: a general appraisal and potential of lactic acid bacteria as biopreservatives. International Research Journal of Biotechnology, 3: 033-046.

Sahnouni F, Boutiba-Maatallah A, Bouhadi D, Boutiba Z. 2014. Characterization of bacteriocin produced by Lactococcus lactis ssp. lactis strains isolated from marine fish caught in the Algerian west coast. Turkish Journal of Agricultural and Natural Sciences, 2: 1138-1143. 